\title{
The Lower Prolactin Serum Level Is a Characteristic of Anorexia Nervosa with Depression
}

\author{
Jihui Yue*, Minfeng Cheng*, Hong Wang, Shenglin Wen\# \\ Department of Psychology, Fifth Affiliated Hospital, Sun Yat-sen University, Zhuhai, China \\ Email: "wenshl@mail.sysu.edu.cn
}

How to cite this paper: Yue, J. H., Cheng, M. F., Wang, H., \& Wen, S. L. (2019). The Lower Prolactin Serum Level Is a Characteristic of Anorexia Nervosa with Depression. Psychology, 10, 1615-1621.

https://doi.org/10.4236/psych.2019.1012106

Received: May 19, 2019

Accepted: September 17, 2019

Published: September 20, 2019

Copyright (๑) 2019 by author(s) and Scientific Research Publishing Inc. This work is licensed under the Creative Commons Attribution International License (CC BY 4.0).

http://creativecommons.org/licenses/by/4.0/ (c) (i) Open Access

\begin{abstract}
This study was designed to explore the relationship between anorexia nervosa and depression and to evaluate the role that endocrine hormones play in anorexia nervosa with functional hypothalamic amenorrhea and comorbid depression. In this study, thyroid hormones and sex hormones were compared between 30 first-episode drug-naive female patients with anorexia nervosa with depression (AND), and 20 female patients with anorexia nervosa with no depression (ANND). There were no differences between anorexia nervosa participants with and without depression in thyroid hormones levels. Patients with AND had significantly lower prolactin serum levels; patients with AND had a median $\left[\mathrm{P}_{25}, \mathrm{P}_{75}\right]$ prolactin level of $213.0 \mathrm{uIU} / \mathrm{ml}[12.1$ 268.2] vs. $446.6 \mathrm{uIU} / \mathrm{ml}$ [119.1 - 635.5] for patients with ANND. There were no differences in other sex hormones between the two groups. Our data suggested that a lower prolactin serum level is a characteristic of first-episode neuroleptic-naive patients with AND.
\end{abstract}

\section{Keywords}

Anorexia Nervosa, Prolactin, Depression, Amenorrhea, Body Mass Index

\section{Introduction}

Anorexia nervosa (AN) is a potentially life-threatening disorder typically seen in adolescent girls (Stergioti et al., 2013). Its diagnosis primarily depends on the clinical manifestation, which is characterized by a serious disturbance in eating behavior, along with cognitive distortions about body shape or body weight. Serious medical complications affect almost every organ system including electro-

*Jihui Yue and Minfeng Cheng contributed equally to this paper. 
lyte disorders, hematologic disorders, endocrine changes, and severe bone loss (American Psychiatric Association, 2013). Although the disorder has been studied from the multifactorial model to a complex developmental model (Garner, 1993; Kaye et al., 2009; Herpertz-Dahlmann et al., 1997), the etiology of AN remains elusive. AN is associated with a lifelong trajectory and the continued appearance of multiple risk factors over the entire disease time course (Gicquel, 2013).

Adolescents with functional hypothalamic amenorrhea show a higher incidence of subclinical symptoms of AN, including psychosomatic discomfort, and mild depressive traits (Bomba et al., 2007). Functional hypothalamic amenorrhea might represent part of the AN biological spectrum, and a link between these two conditions might exist, possibly related to the degree of psychological and/or hormonal dysfunction (Bomba et al., 2014). Elevated transaminases are common in severely malnourished patients with AN (Hanachia et al., 2013). In addition, with the exception of the reports, four risk factors including young age, low body mass index (BMI), the pure restrictive form of the disease, and male sex were observed.

The presence of suicidal thoughts and self-injurious behaviors in patients with eating disorders is well known. A significant percentage of adolescents with eating disorders present negative emotions and suicidal ideation (Varela-Besteiro et al., 2017). Self-criticism is a significant problem in depression and eating disorders (Thew et al., 2017).

The limitations of studies on patients with $\mathrm{AN}$ and comorbid depression have received little attention. Therefore, we performed this study to evaluate endocrine hormones levels in the presence of these conditions in an attempt to further explore the relationship between $\mathrm{AN}$ and depression and to evaluate the role that endocrine hormones play in AN and comorbid depression.

\section{Materials and Methods}

First-episode inpatients $(\mathrm{n}=50)$ with AN were recruited from inpatient units of the Third Affiliated Hospital of Sun Yat-sen University, Guangzhou, Guangdong Province, PR China. Most patients were hospitalized for diagnostic or therapeutic purposes for endocrinology-related symptoms. All subjects were adolescent girls presented with severely malnourish and functional hypothalamic amenorrhea. Ethical written informed consent was obtained from all patients in accordance with protocols approved by the Clinical Research Ethics Committee of the Third Affiliated Hospital of Sun Yat-sen University.

A total of 50 first-episode patients presented with AND; the patients were diagnosed according to the DSM-IV criteria. The patients were divided into two groups based on the presence of comorbid depression: 30 patients with AND, and 20 patients with ANND. A chemiluminescence immunoassay (SIEMENS Advia Centaur XP) was used to measure endocrine hormones levels prior to any medication after hospitalization. Endocrine hormones, including free triiodo- 
thyronine, free unbound thyroxine, thyroid stimulating hormone, prolactin, follicle-stimulating hormone, luteinizing hormone, and estradiol were recorded at admission prior to any medication after hospitalization.

The exclusion criteria for all subjects were as follows: 1) depression that was clearly due to substance abuse, and any other type of psychiatric disturbance; 2) a history of antipsychotic therapy or other medication; 3) physical illnesses including any with cerebral or endocrine pathology; and 4) premenopausal or pregnant women.

All statistical analyses were conducted with Statistical Program for the Social Sciences (SPSS) software (version 22.0, Chicago, IL, USA). Comparisons between the AN and AND groups were evaluated by Student's t-test or Mann-Whitney test. The statistical significance was set at $p<0.05$.

\section{Results}

The two groups were similar with regard to age, body mass index, and duration of AN (see Table 1). There were no differences between anorexia nervosa participants with and without depression in thyroid hormones levels.

The two groups differed with regard to prolactin (see Table 1 and Figure 1). AND patients had a median $\left[\mathrm{P}_{25}, \mathrm{P}_{75}\right]$ prolactin level of $213.0 \mathrm{uIU} / \mathrm{mL}[12.1$ $268.2]$ vs. $446.6 \mathrm{uIU} / \mathrm{mL}$ [119.1 - 635.5] for Group ANND patients $(p=0.004)$. There were no differences in other sex hormones between the two groups.

\section{Discussion}

In our study, $60 \%$ of the participants showed depression. Patients with AN often report feeling markedly less well, and this may be associated with changes in

Table 1. Demographic and endocrine hormones values at admission.

\begin{tabular}{|c|c|c|c|}
\hline & ANND $(n=20)$ & $\operatorname{AND}(\mathrm{n}=30)$ & $p$ value \\
\hline Mean age $( \pm S D)$ & $16.2(3.1)$ & $15.5(2.0)$ & 0.267 \\
\hline Body mass index $( \pm S D)$ & $12.6(1.7)$ & $13.3(1.8)$ & 0.083 \\
\hline Duration of $\mathrm{AN}^{\mathrm{a}}\left(\mathrm{P}_{25}, \mathrm{P}_{75}\right)$ & $7(6-12)$ & $8(6-12)$ & 0.299 \\
\hline Free triiodothyronine $\mathrm{e}^{\mathrm{b}}( \pm \mathrm{SD})$ & $2.9(0.8)$ & $2.9(0.7)$ & 0.716 \\
\hline Free unbound thyroxine $e^{c}( \pm S D)$ & $13.8(2.5)$ & $12.9(2.4)$ & 0.215 \\
\hline Thyroid stimulating hormone $( \pm \mathrm{SD})$ & $1.5(0.8)$ & $1.8(1.0)$ & 0.190 \\
\hline $\operatorname{Prolactin}^{\mathrm{e}}\left(\mathrm{P}_{25}, \mathrm{P}_{75}\right)$ & $446.6(119.1-635.5)$ & $213.0(12.1-268.2)$ & 0.004 \\
\hline Follicle stimulating hormone $\mathrm{f}^{\mathrm{f}}\left(\mathrm{P}_{25}, \mathrm{P}_{75}\right)$ & $0.5(0.0-1.8)$ & $0.7(0.2-2.0)$ & 0.437 \\
\hline Luteinizing hormone $\mathrm{e}^{\mathrm{g}}\left(\mathrm{P}_{25}, \mathrm{P}_{75}\right)$ & $0.0(0.0-0.1)$ & $0.0(0.0-0.4)$ & 0.558 \\
\hline Estradiol $^{\mathrm{h}}\left(\mathrm{P}_{25}, \mathrm{P}_{75}\right)$ & $15.9(6.6-106.6)$ & $29.8(20.9-86.4)$ & 0.735 \\
\hline
\end{tabular}

${ }^{a}$ Duration of AN given in months. ${ }^{b}$ Reference free triiodothyronine serum level: 3.10 - $6.80 \mathrm{pmol} / \mathrm{L}$. ${ }^{\mathrm{c}} \mathrm{Refe}$ rence free unbound thyroxine serum level: $12.30-20.20 \mathrm{pmol} / \mathrm{L}$. ${ }^{\mathrm{d}}$ Reference thyroid stimulating hormone serum level: 0.27 - $4.20 \mathrm{uIU} / \mathrm{mL}$. ${ }^{\mathrm{e}}$ Reference prolactin serum level, women who are menopausal: 59 - 619 $\mathrm{uIU} / \mathrm{mL}$. ${ }^{\mathrm{f}}$ Reference follicle stimulating hormone serum levels, women who are menopausal: $26.72-133.41$ $\mathrm{mIU} / \mathrm{mL}$. ${ }^{g}$ Reference luteinizing hormone serum levels, women who are menopausal: $1.78-92.10 \mathrm{mIU} / \mathrm{mL}$. ${ }^{\mathrm{h}}$ Reference estradiol serum levels, women who are menopausal: $<10-28 \mathrm{pg} / \mathrm{mL}$. 


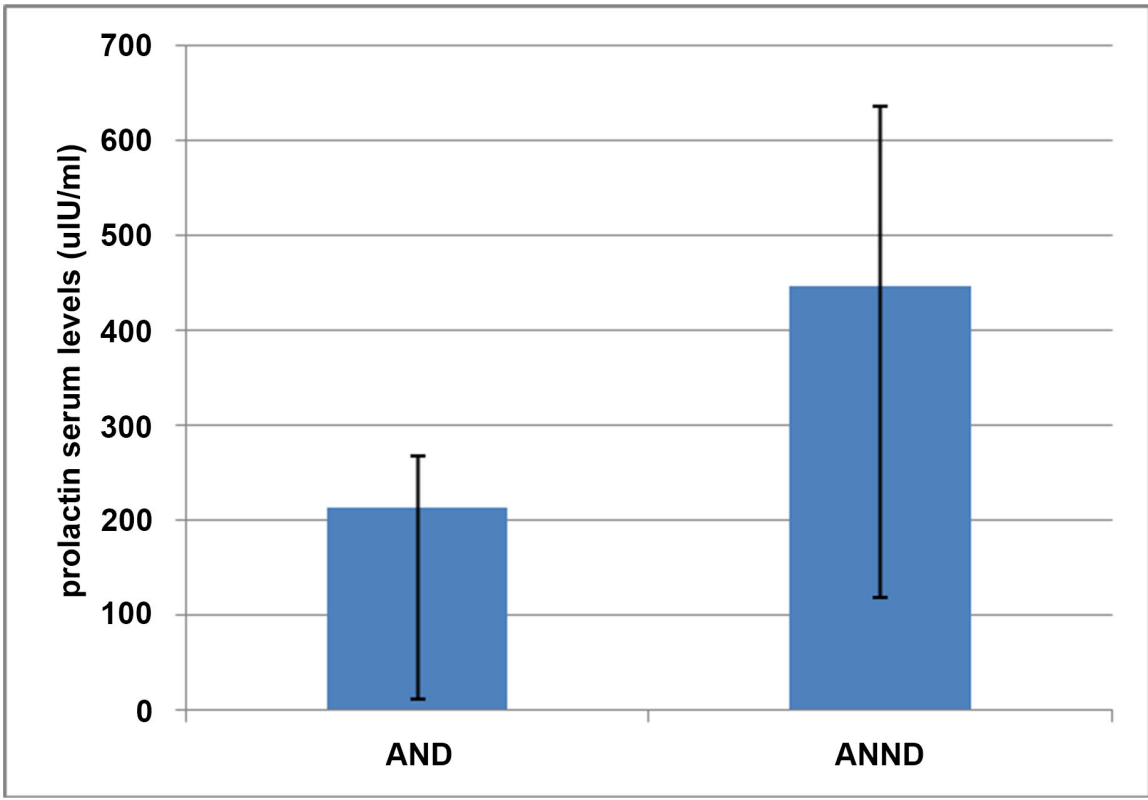

Figure 1. Distribution of serum prolactin levels in two groups: The horizontal line within the colored box represents the median. The data between the colored box and the tails contain the upper and lower quartiles. Reference prolactin serum level, women who are not premenopausal or pregnant: $59-619 \mathrm{uIU} / \mathrm{ml}$.

steroid hormones (Rigaud et al., 2010; Wassif et al., 2011). Individuals with AN have been observed to have lower levels of emotion expression (Davies et al., 2011; Rhind et al., 2014). Age was not associated with poor emotion expression, but characteristics involving lower BMI, social anhedonia, and depression were associated with poor emotion expression (Lang et al., 2016). The evidence for depression in patients with AN associated with increased levels of pro-inflammatory mediators has been less clear. Metabolic disturbances in AN would lead to a pattern of immune disturbances distinct from that of depression. Loss of adipose tissue may attenuate cytokine production and thus modulate the experience of illness (Pisetsky et al., 2014). The AN subjects had low concentrations of insulin-like growth factor-1, relative hypercortisolemia, leptin, insulin, amylin, and incretins; and high concentrations of ghrelin, peptide YY, and adiponectin. These changes might affect neurocognition, anxiety, depression, and the psychopathology of AN (Misra \& Klibanski, 2014).

In this study, all subjects were adolescent girls who presented with severe malnourishment and functional hypothalamic amenorrhea. The data on the hormonal functions are intriguing. Low levels of luteinizing hormone and follicle-stimulating hormone at baseline remained low at 1-year follow-up in AN subjects; and, resumption of menses did not depend on BMI but on restoration of hypothalamic-pituitary-ovarian function (Golden et al., 1997). We can assume that hormonal imbalance resulted from an altered hypothalamic-pituitary tone secondary to aforementioned structural brain changes and hypothalamic-pituitary-ovarian dysfunction associated with worsened malnutrition. 
We found significant differences in prolactin serum levels between AND and ANND patients at admission. Patients with AND had lower prolactin serum levels than those in the ANND group. The results showed that decreased prolactin serum levels, which were caused by depression, were related to emotion. Research shows that prolactin can affect the dopaminergic activity (DA) and change the DA receptor sensitivity (Aihara et al., 2004). The results indicate that the difference in serum prolactin levels between groups could result from the specific pattern of prolactin secretion with alterations in DA. It was not a change in BMI but depression that played a role in treatment outcomes of patients with eating disorders (Fewell et al., 2017). Therefore, alleviating a depressive symptom could be considered as a first-line treatment option for patients with AN with depression.

Our study is limited by an inevitable selection bias because the study sample consisted of patients from our hospital. In addition, the study group consisted only of females, and there was a lack of clinical data after treatment. Such a sample may not be representative of all patients with AN with depression. In addition, this study did not include a depression control group; we cannot conclude that reduced prolactin serum levels are specific to AN or apparent in depression disorders in general. The results must be confirmed in a multicenter study before definite conclusions can be drawn.

\section{Conclusion}

In summary, this study included adolescent girls who presented with severe malnourishment and functional hypothalamic amenorrhea. Patients with AND had lower prolactin serum levels than those patients with ANND. A better understanding of clinical characteristics of AN with depression would provide better treatment for patients with these problems.

\section{Contributors}

Wen Shenglin designed the study. Yue Jihui and Wang Hong collected the original data. Cheng Minfeng managed and analyzed the data. Yue Jihui wrote the manuscript. Wen Shenglin revised the paper. All authors contributed to and have approved the final manuscript.

\section{Acknowledgements}

The study was supported by grants from the Technology Project of Guangdong Province, China (No. 2014A020212530).

\section{Conflicts of Interest}

No conflict of interest declared.

\section{References}

Aihara, K., Shimada, J., Miwa, T., Tottori, K., Burris, K. D., Yocca, F. D., Horie, M., \& 
Kikuchi, T. (2004). The Novel Antipsychotic Aripiprazole Is a Partial Agonist at Short and Long Isoforms of $\mathrm{D}_{2}$ Receptors Linked to the Regulation of Adenylyl Cyclase Activity and Prolactin Release. Brain Research, 1003, 9-17.

https://doi.org/10.1016/j.brainres.2003.09.082

American Psychiatric Association (2013). Diagnostic and Statistical Manual of Mental Disorders: DSM-5 (5th ed.). Arlington, VA: American Psychiatric Association. https://doi.org/10.1176/appi.books.9780890425596

Bomba, M., Corbetta, F., Gambera, A., Nicosia, F., Bonini, L., Neri, F., Tremolizzo, L., \& Nacinovich, R. (2014). Heart Rate Variability in Adolescents with Functional Hypothalamic Amenorrhea and Anorexia Nervosa. Psychiatry Research, 215, 406-409. https://doi.org/10.1016/j.psychres.2013.11.012

Bomba, M., Gambera, A., Bonini, L., Peroni, M., Neri, F., Scagliola, P., \& Nacinovich, R. (2007). Endocrine Profiles and Neuropsychologic Correlates of Functional Hypothalamic Amenorrhea in Adolescents. Fertility and Sterility, 87, 876-885.

https://doi.org/10.1016/j.fertnstert.2006.09.011

Davies, H., Schmidt, U., Stahl, D., \& Tchanturia, K. (2011). Evoked Facial Emotional Expression and Emotional Experience in People with Anorexia Nervosa. International Journal of Eating Disorders, 44, 531-539. https://doi.org/10.1002/eat.20852

Fewell, L. K., Levinson, C. A., \& Stark, L. E. (2017). Depression, Worry, and Psychosocial Functioning Predict Eating Disorder Treatment Outcomes in a Residential and Partial Hospitalization Setting. Eating and Weight Disorders, 22, 291-301. https://doi.org/10.1007/s40519-016-0357-6

Garner, D. M. (1993). Pathogenesis of Anorexia Nervosa. The Lancet, 341, 1631-1635. https://doi.org/10.1016/0140-6736(93)90768-C

Gicquel, L. (2013). Anorexia Nervosa during Adolescence and Young Adulthood: Towards a Developmental and Integrative Approach Sensitive to Time Course. Journal of Physiology-Paris, 107, 268-277. https://doi.org/10.1016/j.jphysparis.2013.03.010

Golden, N. H., Jacobson, M. S., Schebendach, J., Solanto, M. V., Hertz, S. M., \& Shenker, I. R. (1997). Resumption of Menses in Anorexia Nervosa. Archives of Pediatrics and Adolescent Medicine, 151, 16-21. https://doi.org/10.1001/archpedi.1997.02170380020003

Hanachia, M., Melchior, J. C., \& Crenn, P. X. (2013). Hypertransaminasemia in Severely Malnourished Adult Anorexia Nervosa Patients: Risk Factors and Evolution under Enteral Nutrition. Clinical Nutrition, 32, 391-395.

https://doi.org/10.1016/j.clnu.2012.08.020

Herpertz-Dahlmann, B., Seitz, J., \& Konrad, K. X. (1997). Aetiology of Anorexia Nervosa: from a "Psychosomatic Family Model" to a Neuropsychiatric Disorder? European Archives of Psychiatry and Clinical Neuroscience, 261, 177-181.

https://doi.org/10.1007/s00406-011-0246-y

Kaye, W. H., Fudge, J. L., \& Paulus, M. (2009). New Insights into Symptoms and Neurocircuit Function of Anorexia Nervosa. Nature Reviews Neuroscience, 10, 573-584. https://doi.org/10.1038/nrn2682

Lang, K., Larsson, E. E. C., Mavromara, L., Simic, M., Treasure, J., \& Tchanturia, K. (2016). Diminished Facial Emotion Expression and Associated Clinical Characteristics in Anorexia Nervosa. Psychiatry Research, 236, 165-172.

https://doi.org/10.1016/j.psychres.2015.12.004

Misra, M., \& Klibanski, A. (2014). Endocrine Consequences of Anorexia Nervosa. The Lancet Diabetes and Endocrinology, 2, 581-592. https://doi.org/10.1016/S2213-8587(13)70180-3 
Pisetsky, D. S., Trace, S. E., Brownley, K. A., Hamer, R. M., Zucker, N. L., Roux-Lombard, P., Dayer, J.-M., \& Bulik, C. M. (2014). The Expression of Cytokines and Chemokines in the Blood of Patients with Severe Weight Loss from Anorexia Nervosa: An Exploratory Study. Cytokine, 69, 110-115. https://doi.org/10.1016/j.cyto.2014.05.018

Rhind, C., Mandy, W., Treasure, J., \& Tchanturia, K. (2014). An Exploratory Study of Evoked Facial Affect in Adolescent Females with Anorexia Nervosa. Psychiatry Research, 220, 711-715. https://doi.org/10.1016/j.psychres.2014.07.057

Rigaud, D., Boulier, A., Tallonneau, I., Brindisi, M. C., \& Rozen, R. (2010). Body Fluid Retention and Body Weight Change in Anorexia Nervosa Patients during Refeeding. Clinical Nutrition, 29, 749-755. https://doi.org/10.1016/j.clnu.2010.05.007

Stergioti, E., Deligeoroglou, E., Economou, E. et al. (2013). Gene Receptor Polymorphism as a Risk Factor for BMD Deterioration in Adolescent Girls with Anorexia Nervosa. Gynecological Endocrinology, 29, 716-719. https://doi.org/10.3109/09513590.2013.798275

Thew, G. R., Gregory, J. D., Roberts, K., \& Rimes, K. A. (2017). Self-Critical Thinking and Overgeneralization in Depression and Eating Disorders: An Experimental Study. Behavioural and Cognitive Psychotherapy, 45, 510-523.

Varela-Besteiro, O., Serrano-Troncoso, E., Rodríguez-Vicente, V., Curet-Santisteban, M., Conangla-Roselló, G., Cecilia-Costa, R., Carulla-Roig, M., Matalí-Costa, J. L., \& Dolz-Abadia, M. (2017). Suicidal Ideation and Self-Injurious Behavior in Adolescents with Eating Disorders. Actas Españolas de Psiquiatría, 45, 157-166.

Wassif, W. S., McLoughlin, D. M., Vincent, R. P., Conroy, S., Russell, G. F. M., \& Taylor, N. F. (2011). Steroid Metabolism and Excretion in Severe Anorexia Nervosa: Effects of Refeeding. The American Journal of Clinical Nutrition, 93, 911-917. https://doi.org/10.3945/ajcn.111.012666 